\title{
Treinamento de Habilidades Sociais: Avaliação de um Programa de Desenvolvimento Interpessoal Profissional para Universitários de Ciências Exatas
}

\author{
Daniele Carolina Lopes \\ Denise Dascanio \\ Bárbara Carvalho Ferreira \\ Zilda Aparecida Pereira Del Prette \\ Almir Del Prette
}

\section{RESUMO}

A valorização das relações interpessoais no trabalho tem trazido novas demandas para a formação profissional universitária, especificamente, em relação ao ensino e desenvolvimento de habilidades interpessoais aliadas às habilidades acadêmicas e técnicas. Este estudo avaliou um programa de treinamento de habilidades sociais com universitários de cursos de Ciências Exatas. Participaram 35 estudantes, sendo 23 homens e 12 mulheres, distribuídos em três diferentes grupos consecutivos de intervenção. A avaliação do repertório de habilidades sociais foi realizada com o Inventário de Habilidades sociais (IHS), antes, em uma sessão intermediária, imediatamente após o programa e três meses ao término da intervenção. Tanto nas análises de grupo como nas individuais foi possível observar aquisições em habilidades sociais e manutenção delas, especialmente no Escore Total e nas subescalas F1 - Enfrentamento com risco e F4 - Autoexposição a desconhecidos ou situações novas. Os resultados indicaram eficácia e efetividade de um programa de desenvolvimento profissional interpessoal para universitários em transição para o mercado de trabalho e sugere novas questões de pesquisa.

Palavras-chaves: treinamento de habilidades sociais; relações de trabalho; universitários; ciências exatas.

\section{ABSTRACT \\ Social Skills Training: Evaluation of an Interpersonal Professional Development Program for undergraduates of Exact Sciences courses}

The importance of interpersonal work relationships has brought new demands for the professional development of college students, specifically, in relation to the teaching and development of interpersonal skills coupled with academic and technical skills. This study evaluated a social skills training program with students from Exact Sciences careers. Participants were 35 undergraduates, 23 males and 12 females, distributed in three different groups of consecutive interventions. The students were evaluated with Social Skills Inventory (IHS) before, in a intermediate session, immediately after and three months after the intervention program. The analysis in group and individually showed significant acquisitions in social skills and their maintenance, especially in the total score and F1 - Self-affirmation when coping with risk and F4 - Exposure of self with strangers and in new situations subscales of social skills. These results indicated the efficiency and effectiveness of an interpersonal professional development program for undergraduates in transition to labor market and suggest new research questions.

Keywords: social skills training; work relationship; undergraduates; exact sciences careers.

\section{Sobre os Autores}

D.C.L.

orcid.org/0000-0002-3578-8606 Universidade Federal de São Carlos - São Carlos, SP danielecalopes@hotmail.com

D.D.

orcid.org/0000-0002-0790-2504

Unip - Bauru - Bauru, SP

denisedascanio@yahoo.com.br

\section{B.C.F.}

orcid.org/0000-0002-9759-5682 Universidade Federal dos Vales do Jequitinhonha e Mucuri UFVJM/Campus Diamantina Diamantina, MG

barbaracarvalho_ufvjm@yahoo.com.br

\section{Z.A.P.P.}

orcid.org/0000-0002-0130-2911 Universidade Federal de São Carlos, Instituto Nacional de Ciência e Tecnologia (INCTECCE) - São Carlos, SP zdprette@ufscar.br

\section{A.D.P.}

orcid.org/0000-0003-2051-1214 Universidade Federal de São Carlos - São Carlos, SP adprette@ufscar.br

\section{Direitos Autorais}

Este é um artigo de acesso aberto e pode ser reproduzido livremente, distribuído, transmitido ou modificado, por qualquer pessoa desde que usado sem fins comerciais. 0 trabalho é disponibilizado sob a licença Creative Commons CCBY-NC. 


\section{-4* INTERACÃO EM L* PSICOLOGIA}

0 rápido processo de mudança das organizações (International Labour Organization - ILO, 2006) como fruto da globalização, do mercado, da competitividade, das novas matérias-primas, procedimentos e produtos, tem inúmeras implicações no âmbito econômico, social, cultural, político e tecnológico. Para as organizações, tais mudanças implicam reestruturação em seus processos e funcionamento para atender às novas competências e exigências que, por sua vez, afetam o perfil profissiográfico do trabalhador (MorenoJimenez \& Camacho, 2014). Nesse cenário, Moreno-Jimenez e Camacho (2014) destacaram que somente a competência técnica e o esforço não são suficientes para lidar com essas demandas, dando destaque a novas competências, principalmente, as interpessoais e sociais.

As consequências geradas pela globalização (novo mercado, competitividade, novas matérias-primas, procedimentos e produtos) afetaram as formas de produção, que passaram a se centrar na prestação de serviços e a exigir alterações nas relações laborais, sociais, informais, além de melhoria na qualidade dos contatos interpessoais, inclusive para lidar com a diversidade étnica e cultural com a incorporação dos estrangeiros (Gil, 1998; Marqués, Farrerons, Arian, \& Quiroga, 2012; Moreno-Jimenez \& Camacho, 2014). Segundo revisão de literatura realizada por Silva e Teixeira (2012), algumas competências que contribuem para uma atuação eficaz, em diferentes situações do ambiente profissional, são a capacidade de trabalho em equipe, a responsabilidade e a comunicação. Para esses autores, tais competências transversais "são elementos que favorecem o bom desempenho no mundo do trabalho, uma vez que ajudam os sujeitos a lidarem melhor com diferentes tipos de situações laborais e facilitam a aplicação das suas competências técnicas" (p. 200). Assim, além de habilidades técnicas, tornaram-se mais valorizadas as competências para trabalhar em equipe, falar em público, resolver problemas, tomar decisões, comunicar-se de forma empática e assertiva, entre outras.

A importância das habilidades interpessoais é cada vez mais aceita no âmbito das organizações. Uma evidência disso é o recente projeto europeu denominado "Europa, 2020" que tem por objetivo de transformar a União Europeia em uma economia sustentável geradora de emprego, produtividade e desenvolvimento a partir de organizações saudáveis), cuja proposta prevê o desenvolvimento de habilidades e competência sociais e interpessoais como forma de garantir flexibilidade, eficiência corporativa, bem-estar subjetivo e saúde dos trabalhadores (Moreno-Jiménez, Garrosa \& RodríguezCarvajal, 2013).

Em relação à formação inicial do indivíduo no Ensino Superior, há uma discussão, ainda que mais presente na Psicologia, a respeito da importância de capacitar/formar
Daniele Carolina Lopes, Denise Dascanio, Bárbara Carvalho Ferreira, Zilda Aparecida Pereira Del Prette e Almir Del Prette profissionais preparados para lidar com o meio físico e social de suas profissões e sobre como vem sendo realizada a capacitação nos cursos de graduação pela Universidade (Botomé, 2010; Kienen, Kubo \& Botomé, 2013; Kubo \& Botomé, 2003). A respeito da capacitação interpessoal, a Universidade ainda precisa assumir um papel mais ativo na formação de profissionais, de modo a atender às novas demandas do mercado de trabalho. Não obstante, algumas universidades têm demonstrado maior sensibilidade nessa direção, investindo na organização de estágios, empresas "juniores", programas de formação continuada e projetos de extensão universidadeempresa (A. Del Prette \& Del Prette, 2003; Mourão, Puente-Palacios, 2006). Nessa direção, vem sendo reconhecida a insuficiência de estratégias e a importância de garantir o desenvolvimento social e emocional de futuros profissionais na universidade (Fang, 2012; Gerolamo \& Gambi, 2013; Gondim, 2002; Jablokow, 2008; Ponsa, Manresa-Yee, Arellano, Gomez, \& Perez, 2012).

Em resumo, pode-se defender que o perfil requerido dos profissionais egressos ou não da Universidade, necessariamente, inclui um conjunto de habilidades relacionadas ao desempenho interpessoal. Essas habilidades constituem o objeto de um campo designado, na Psicologia, de Habilidades Sociais (HS). No contexto de trabalho, esse perfil remete, mais especificamente, às chamadas habilidades sociais profissionais, definidas como "aquelas que atendem às diferentes demandas interpessoais do ambiente de trabalho objetivando o cumprimento de metas, a preservação do bem-estar da equipe e o respeito aos direitos de cada um" (A. Del Prette \& Del Prette, 2001, p. 89).

A preocupação com a promoção de habilidades sociais de universitários, particularmente de habilidades sociais profissionais, aparece de forma pioneira nos trabalhos de Argyle (1967/1994). No Brasil, já na década de 1970, Del Prette (1978) relata um treino assertivo com estudantes de Psicologia, destacando a importância de se promover habilidades sociais para o exercício profissional. Além de conduzirem programas de Treinamento de Habilidade Sociais (THS) com estudantes de Psicologia (Del Prette, Del Prette \& Barreto, 1999; A. Del Prette \& Del Prette, 2003), Z. Del Prette e Del Prette (2003), em um ensaio temático, discutiram a importância do desenvolvimento interpessoal no Ensino Superior como uma questão ainda pendente na formação universitária, com impacto para a saúde e o funcionamento psicossocial.

Conforme estudo bibliométrico de Prytz Nilsson, Suarez e Olaz (2009), a população de universitários, no período de 1970 a 2008, foi a terceira mais estudada na América Latina, ficando atrás apenas de crianças e sujeitos clínicos. No entanto, em termos de intervenção, a revisão de Bolsoni-Silva et al., (2006), cobrindo toda a produção nacional em periódicos 


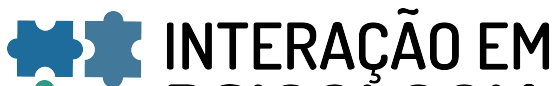 L* PSICOLOGIA}

Daniele Carolina Lopes, Denise Dascanio, Bárbara Carvalho Ferreira Zilda Aparecida Pereira Del Prette e Almir Del Prette indexados até 2004, mostrou que, dos 11 estudos sobre universitários, apenas um envolvia avaliação de programa de intervenção publicado no país: o de Falcone (1999), voltado para a promoção de habilidades sociais empáticas com estudantes de diversos cursos. Nesse mesmo ano, o artigo Del Prette et al., (1999), publicado no exterior, descrevia um estudo quase-experimental de intervenção em habilidades sociais, denominado de Programa de Desenvolvimento Interpessoal Profissional (PRODIP), com resultados sugestivos de sua eficácia e potencialidade no desenvolvimento interpessoal em estudantes de Psicologia.

Além desses dois estudos, novos programas de THS foram depois conduzidos com estudantes de Psicologia (Magalhães, \& Murta, 2003; Pacheco \& Rangé, 2006) e de outras áreas, dentre os quais podem ser citados: (a) o estudo piloto com quatro graduandos de diferentes cursos que apresentavam dificuldades interpessoais (Boas, Silveira \& Bolsoni-Silva, 2005); (b) o programa PRODIP com estudantes de Ciências Exatas (A. Del Prette \& Del Prette, 2003); (c) o programa com universitários e recém-formados de vários cursos (Bolsoni-Silva, Leme, Lima, Costa-Júnior \& Correia, 2009); (d) e o programa de empatia com estudantes de enfermagem (Kestenberg \& Falcone, 2011).

Dentre os estudos sobre habilidades sociais de universitários que, não envolveram intervenção (em geral descritivos e correlacionais), alguns focalizaram graduandos de Psicologia (Carneiro \& Teixeira, 2011; Del Prette, Del Prette, Barreto et al., 2004; Del Prette, Del Prette, Ríos-Saldanha et al., 2004; Gomes \& Soares, 2013, Soares, Poubel \& Mello, 2009) e outros de diversas áreas de formação (Bandeira \& Quaglia, 2005; BolsoniSilva, Loureiro, Rosa \& Oliveira, 2010; Furtado, Falcone \& Clark, 2003).

Esses estudos identificaram déficits de habilidades sociais dos estudantes que justificariam intervenções do tipo PRODIP. $O$ estudo com graduandos de Medicina de Furtado et al. (2003) encontrou relação entre déficits em habilidades sociais e indicadores de estresse. Bandeira e Quaglia (2005) caracterizaram as situações sociais - incômodas ou desagradáveis e cômodas ou agradáveis - de universitários de Ciências Humanas e Exatas, destacando as que envolviam expressar insatisfação e/ou solicitar mudança de comportamento. Bolsoni-Silva et al, (2010) caracterizaram o repertório de habilidades sociais de estudantes de um curso de Desenho Industrial, indicando que os homens apresentaram repertório mais elaborado que as mulheres ainda que, na análise funcional realizada a partir das descrições das respostas a um questionário, as mulheres tenham se mostrado mais competentes na habilidade de falar para público desconhecido, fazer críticas aos colegas e amigos e expressar sentimentos positivos aos namorados. Em outro estudo, com 74 estudantes universitários de 17 cursos diferentes, Ribeiro e Bolsoni-Silva (2011) constataram que esses apresentavam dificuldades no contexto da faculdade/trabalho, como, por exemplo, expor-se em público, expressar opiniões, lidar com crítica, ou seja, "comportamentos de comunicar-se, expressar-se, resolver conflitos e solicitar mudança de comportamento" (p. 217). A partir de suas pesquisas, Bolsoni-Silva e Loureiro (2016) validaram um instrumento para avaliação das habilidades sociais, comportamentos e contextos para universitários. Todos esses estudos, com universitários de cursos que não o de Psicologia, apontaram dificuldades em interações sociais e ilustraram a importância das habilidades sociais para a qualidade das interações, para o enfrentamento de situações adversas do cotidiano e para situações específicas requeridas no ambiente profissional.

O conjunto de estudos de caracterização e de intervenção, antes referidos, mostra a existência de dificuldades interpessoais em universitários, de diferentes cursos e áreas de formação, e apresenta evidências que tais dificuldades podem ser amenizadas ou superadas por meio de condições estruturadas de ensino. De forma indireta, também evidenciam que, salvo experiências ainda esparsas, a formação universitária ainda não articula, de forma suficiente e efetiva, a promoção das competências técnicas ao desenvolvimento socioemocional dos estudantes nas disciplinas e práticas acadêmicas, não obstante a reconhecida necessidade de aprimorar o repertório social em praticamente todas as áreas de atuação e as evidências de dificuldades interpessoais desses estudantes. Na formação universitária, essa carência pode ser ainda mais crítica nas áreas de exatas e tecnológicas por tradicionalmente, não contemplarem esses temas, mais comuns nas ciências humanas.

Um exemplo de articulação entre a formação técnica e interpessoal na graduação de Ciências Exatas foi enfatizada por Liebenberg e Mathews (2012), que defenderam uma reestruturação curricular dos cursos de Engenharia para responder às novas demandas do mercado. Esse estudo ilustra uma experiência bem-sucedida de inclusão de atividades curriculares baseadas na multidisciplinaridade, trabalho em equipe e aprimoramento das habilidades sociais, na Universidade de Pretória (África do Sul). A lacuna em formação interpessoal e socioemocional foi evidenciada por Borchardt, Vaccaro, Azevedo e Ponte Jr. (2009) ao consultarem gestores de empresas, de uma região brasileira, a respeito de competências relevantes para engenheiros. Os autores encontraram que uma das competências de maior importância para a organização, referida pelos respondentes, está associada à comunicação e ao relacionamento interpessoal e que esse repertório constitui um diferencial para o sucesso profissional. 


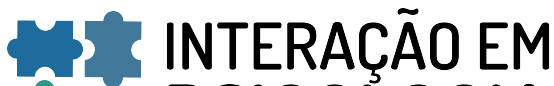 ET PSICOLOGIA}

Considerando a importância das habilidades sociais na formação profissional para lidar com as demandas do trabaIho, principalmente nos cursos com oportunidades escassas na Universidade, como é o caso de estudantes de Ciências Exatas, a proposta de implementação de Programas de Desenvolvimento Interpessoal Profissional (PRODIP) para universitários, tal como testada por Del Prette et al., (1999) e defendida teoricamente (A. Del Prette \& Del Prette, 2001, 2003; Z. Del Prette \& Del Prette, 2003), parece constituir uma alternativa promissora. A proposta do PRODIP se assenta em alguns pressupostos do campo das habilidades sociais, destacados por A. Del Prette e Del Prette (2003): (a) as habilidades sociais são aprendidas ao longo da vida; (b) quando essa aprendizagem não ocorre naturalmente, pode ser planejada por meio de programas especiais de desenvolvimento interpessoal; (c) quando em grupo, os resultados podem ser mais robustos e rápidos; (d) as habilidades devem ser praticadas no ambiente natural; (e) mais do que instruções e preleções, o processo de aprendizagem deve ser baseado no desempenho interativo, tanto no contexto das sessões de grupo como no ambiente natural dos participantes.

O PRODIP se baseia no método vivencial proposto e testado por A. Del Prette e Del Prette (2001; 2003; 2010), entendendo-se as vivências como conjunto de "atividades estruturadas, de modo análogo ou simbólico, a situações cotidianas de interação social dos participantes, que mobiliza sentimentos, pensamentos e ações, com o objetivo de suprir déficits e maximizar habilidades sociais em programas de THS em grupo" (A. Del Prette \& Del Prette, 2001, p. 106). Cada vivência atende a objetivos específicos e, por isso, em cada programa, elas são selecionadas a partir da avaliação dos recursos e déficits interpessoais dos participantes.

Diferentemente de outros estudos de promoção de habilidades sociais com universitários, este estudo buscou aproximar universidade-empresa ao propor uma capacitação profissional interpessoal dentro de um programa de estágio obrigatório da Universidade, articulado com demandas apresentadas pelas empresas contratantes dos estagiários. Essa preocupação vai ao encontro à necessidade de atender às exigências do atual mercado de trabalho e superar a carência, especialmente em cursos de Ciências Exatas, de investimento no desenvolvimento socioemocional dos estudantes.

O objetivo deste estudo foi avaliar o impacto de Programa de Desenvolvimento Interpessoal Profissional (PRODIP) sobre o repertório de habilidades sociais de estudantes universitários de vários cursos de graduação em Ciências Exatas inseridos em um programa de estágio na etapa final da graduação. O PRODIP se configurou como uma das atividades de um programa mais amplo de capacitação para o estágio final obrigatório da graduação, denominado Programa de Ca-
Daniele Carolina Lopes, Denise Dascanio, Bárbara Carvalho Ferreira Zilda Aparecida Pereira Del Prette e Almir Del Prette pacitação em Liderança - PROLIDER (Gerolamo \& Gambi, 2013). Esse programa de capacitação era oferecido e coordenado por um departamento de graduação de uma das universidades em parceria com empresas privadas que, previamente, abriam vagas para estagiários de cursos de Ciências Exatas. O PROLIDER envolvia uma fase de desenvolvimento de habilidades interpessoais (PRODIP) e de liderança no primeiro semestre do ano e uma fase de aquisição de experiência, no segundo semestre, na forma de estágio, dentro da empresa parceira específica (Gerolamo \& Gambi, 2013).

\section{MÉTODO}

\section{PARTICIPANTES}

A amostra foi constituída por 35 estudantes universitários da área de Ciências Exatas que participaram de um PRODIP, em uma de suas três edições anuais consecutivas, compostas, respectivamente, por subgrupos de 16, 10 e nove participantes, sendo todos os subgrupos conduzidos por duas psicólogas, sendo que uma delas esteve presente nas três edições. Dos 35 estudantes, $66 \%(n=23)$ eram do gênero masculino e $34 \%(n=12)$ do feminino, com média de idade de 22,8 anos. Os participantes cursavam o último ano de graduação em Ciências Exatas sendo: Engenharia de Produção $(n=20)$, Engenharia Mecatrônica $(N=6)$, Engenharia da Computação $(n=3)$; Engenharia Elétrica $(n=2)$, Engenharia Aeronáutica $(n=2)$, Ciência da Computação $(n=1)$ e Informática $(n=1)$. Quanto ao nível socioeconômico, obtido pelo Critério Brasil (Índice Brasileiro de Opinião Pública e Estatística IBOPE, 2005) ${ }^{1}$, a composição era predominantemente de classe alta: $A 1=15 ; A 2=11 ; B 1=5$ e $B 2=5$.

\section{AMBIENTE}

Todas as sessões do PRODIP foram conduzidas em uma sala ampla de uma das universidades dos participantes, equipada com cadeiras e equipamentos de áudio e vídeo.

\section{INSTRUMENTOS/MATERIAIS}

Inventário de Habilidades Sociais (IHS-Del-Prette $-Z$. Del Prette \& Del Prette, 2001). Instrumento de autorrelato utilizado para avaliar o repertório de habilidades sociais do indivíduo, constituído de 38 itens, cada um deles apresentando uma situação e reações (socialmente habilidosa ou não) de relacionamento interpessoal em diversos contextos (trabalho, lazer e família) e com diferentes interlocutores (familiares, amigos, autoridade, pessoas desconhecidas). Em cada situação, o respondente deve estimar a freqüência com que reage da forma especificada no item, em uma escala que variam de Nunca ou raramente (0) a Sempre ou quase sempre (4). 0 


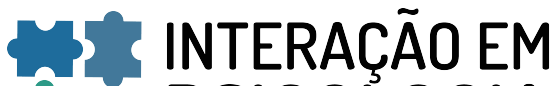 ET PSICOLOGIA}

Daniele Carolina Lopes, Denise Dascanio, Bárbara Carvalho Ferreira Zilda Aparecida Pereira Del Prette e Almir Del Prette
IHS-Del-Prette produz um escore total (ET) e cinco escores fatoriais: F1 - Enfrentamento com risco, F2 - Autoafirmação na expressão do afeto positivo, F3 - Conversação e desenvoltura social, F4 - Autoexposição a desconhecidos ou situações novas e F5 - Autocontrole da agressividade. A análise psicométrica do IHS-Del-Prette, realizada por meio de vários estudos (Del Prette et al., 1998; A. Del Prette, et al., 1999; Bandeira, Costa, Del Prette, Del Prette \& Gerk-Carneiro, 2000), indicou boa consistência interna $(a=0,75)$, estabilidade teste e reteste $(r=0,90, p=0,001)$ e validade concomitante com o Inventário de Rathus $(r=0,79, p=0,01)$.

Diário de campo. Caderno de anotações com o registro assistemático de falas e depoimentos dos participantes em cada sessão, especialmente aquelas indicativas de aquisições ou deficit importantes para o replanejamento do programa.

Materiais de intervenção. Projetor multimídia, vídeos, textos impressos para vivências, além de materiais específicos de acordo com os objetivos de cada vivência sugeridos em A. Del Prette e Del Prette (2001) e/ou previamente planejados pelas psicólogas.

\section{PROCEDIMENTO DE COLETA DE DADOS E INTERVENÇÃO COM O PRODIP}

As avaliações de habilidades sociais dos participantes ocorreram antes, durante (aproximadamente na sétima sessão) e após o programa de intervenção. Com exceção do primeiro grupo, os demais foram alvos de avaliações de seguimento (follow-up), em sessão adicional três meses após o término da intervenção.

A intervenção com o PRODIP teve duração de três meses, com 15 sessões (incluindo as de avaliação), uma por semana, durante o primeiro semestre de cada ano. Cada sessão era norteada por um plano geral que especificava os objetivos comportamentais para os participantes de cada grupo (habilidades sociais mais deficitárias que deveriam ser alvo de atenção para o grupo todo e para participantes específicos) e continha a descrição das atividades e vivências (baseadas em A. Del Prette e Del Prette, 2001) previstas para a intervenção. Esse plano podia ser alterado para atender às novas necessidades detectadas ao longo do programa, permitindo ajustes às características dos participantes. Em geral, porém, foi mantida alta uniformidade entre as três edições do PRODIP.

As sessões iniciais do PRODIP envolviam a promoção de um conjunto de habilidades denominadas "de processo" tais como: observar, descrever, registrar, interpretar, relacionar estímulos antecedentes e consequentes do comportamento, identificar articulações entre pensamentos, sentimentos e comportamentos. Nas sessões intermediárias eram adicionados outros objetivos, alguns comuns ao grupo todo, entre eles a análise e desempenho de comportamentos verbais e não verbais, importantes em habilidades como expressar sentimentos e empatia, dar e receber feedback, elogiar, iniciar e manter conversação. As sessões finais eram destinadas às habilidades consideradas mais complexas e passíveis de gerar ansiedade, como as habilidades assertivas (lidar com críticas, expressar desagrado, aceitar e recusar pedidos, falar com pessoas de autoridade, argumentar, justificar) e de trabalho em grupo (coordenar, cooperar, tomar decisões).

Em termos de procedimento, na primeira sessão, foram apresentados brevemente os objetivos, a estrutura e o funcionamento do programa e, ainda, foi solicitado que cada participante se apresentasse. Nessa sessão foi também solicitada a assinatura do Termo de Consentimento Livre e Esclarecido (para uso dos dados em pesquisa) e aplicado o IHS-Del-Prette (Z. Del Prette \& Del Prette, 2001). Nas demais sessões de intervenção foram realizadas exposições orais dialogadas, nessa temática, com ou sem o uso de projeções de texto e vídeos, e as atividades práticas. A parte prática envolvia a condução de vivências e desempenho de papéis (roleplay), apoiados em técnicas comportamentais como: modelação, modelagem, reforçamento diferencial, instrução, feedback oral e tarefas de casa. Ao final de cada sessão era atribuída uma tarefa interpessoal de casa, procedimento planejado conforme recomendado por A. Del Prette e Del Prette (2005; 2010), visando a diferentes objetivos tais como reconhecer e analisar o próprio desempenho nas diferentes situações interpessoais, bem como ampliar esse desempenho para outros contextos diferentes daqueles aprendidos durante a sessão de intervenção. Além disso, ao final da sessão, pedia-se aos participantes que avaliassem, oralmente ou por escrito, o desempenho próprio e dos colegas, bem como as condições da sessão.

Em resumo, cada sessão foi organizada em uma sequência de etapas: (1) Verificação da tarefa interpessoal de casa; (2) Atividade dialogada e Vivência inicial; (3) Vivência central; (4) Atribuição de nova tarefa interpessoal de casa, que podia ser genérica (a mesma para todos) ou personalizadas (diferenciada de acordo com a necessidade de cada participante); (5) Feedback da sessão. Na última sessão do programa, os participantes foram solicitados a avaliarem, de forma escrita, a contribuição do programa para a sua vida pessoal e profissional.

\section{TRATAMENTO DOS DADOS}

As respostas dos participantes aos itens do IHS-Del-Prette foram convertidas em escores, de acordo com as instruções do manual (Z. Del Prette \& Del Prette, 2001) e dispostas 


\section{W INERAC̄̈OEM ET. PSICOLOGIA}

em planilhas do IBM SPSS 19.0 for Windows. Foram efetuadas análises de variância (ANOVA) para medidas repetidas, com post-hoc Bonferroni. Para os participantes que realizaram avaliação de seguimento foram realizadas análises de comparação entre esses escores e os da pós-intervenção (Teste Wilcoxon, para amostras emparelhadas).

Além dessas análises, os escores pré e pós-intervenção foram plotados em gráficos de dispersão, aplicando-se a análise do Método JT (Jacobson \& Truax, 1991), conforme tutorial desenvolvido por Aguiar, Aguiar e Del Prette (2009), para identificar os participantes que apresentaram mudança estatisticamente confiável, ou seja, acima da variabilidade própria
Daniele Carolina Lopes, Denise Dascanio, Bárbara Carvalho Ferreira, Zilda Aparecida Pereira Del Prette e Almir Del Prette do instrumento (erro de medida). Os relatos dos participantes na sessão final do programa são apresentados como indicadores qualitativos adicionais do impacto do PRODIP.

\section{RESULTADOS}

Na Tabela 1 são apresentadas as médias e os desvios-padrão dos escores dos participantes nas várias avaliações realizadas por meio do IHS-Del-Prette.

Tal como indicado na Tabela 1, de acordo com as análises de variância (ANOVA), foram encontradas diferenças signifi-

Tabela 1. Dados descritivos (média e desvio-padrão) dos escores dos participantes no IHSDel-Prette, na pré-intervenção, avaliação intermediária, pós-intervenção e avaliação de seguimento

\begin{tabular}{|c|c|c|c|c|c|c|}
\hline \multirow{2}{*}{ Indicador } & \multirow{2}{*}{ Variação } & Pré & Inter & Pós & Seg & \multirow{2}{*}{ Diferença } \\
\hline & & $\bar{X}(\mathrm{DP})$ & $\bar{X}(\mathrm{DP})$ & $\bar{X}(\mathrm{DP})$ & $\bar{X}(\mathrm{DP})$ & \\
\hline Total & $0-137$ & $101,8(14,6)$ & $105,4(14,6)$ & $109,8(12,1)$ & $109,8(29,8)$ & Pós>Pré* \\
\hline $\mathrm{F} 1$ & $0-18,2$ & $11,11(2,51)$ & $11,66(2,60)$ & $12,49(2,10)$ & $12,52(3,67)$ & $\begin{array}{l}\text { Pós>Pré* } \\
\text { Pós>Inter* }\end{array}$ \\
\hline $\mathrm{F} 2$ & $0-11,7$ & $8,82(1,51)$ & $8,99(1,18)$ & $9,23(1,32)$ & $9,33(2,6)$ & SD \\
\hline F3 & $0-9,62$ & $7,43(1,50)$ & $7,72(1,25)$ & $7,71(1,16)$ & $7,72(2,28)$ & SD \\
\hline $\mathrm{F} 4$ & $0-5,82$ & $4,29(1,08)$ & $4,56(0,98)$ & $4,78(0,75)$ & $4,65(1,42)$ & Pós>Pré* \\
\hline F5 & $0-2,53$ & $1,42(0,56)$ & $1,33(0,47)$ & $1,30(0,50)$ & $1,15(0,58)$ & SD \\
\hline $\begin{array}{l}\text { lota. } \bar{X}=M \\
\text { tervenção; } \\
\text { la expressã } \\
\text { ituações no }\end{array}$ & $\begin{array}{l}\text {; } \mathrm{DP}=\mathrm{De} \\
=\text { Avaliaçã }\end{array}$ & $\begin{array}{l}\text { io padrão; } \mathrm{Pr} \\
\text { le Seguimento } \\
\text { o; F3 = Conver }\end{array}$ & $\begin{array}{l}=\text { Pré-interven } \\
\text { I = Autoafirma } \\
\text { cão e desenvo }\end{array}$ & $\begin{array}{l}\text {; Inter }=\text { Ava } \\
\text { e enfrentame } \\
\text { a social; F4 = }\end{array}$ & $\begin{array}{l}\text { cõo intermedi } \\
\text { com risco; F2 }\end{array}$ & ; Pós = Pó \\
\hline
\end{tabular}

cativas, indicadoras de aquisições, entre as avaliações pré e pós-intervenção, para o Escore Total de habilidades sociais $[F(2,63)=7,45 ; p=0,002]$, o Fator 1 , Autoafirmação e enfrentamento com risco $[F(2,62)=6,53 ; p=0,004]$ e Fator 4 Autoexposição a desconhecidos e situações novas $[F(2,60)$ =6,78; $p=0,003$.

Ainda no Fator 1 (Autoafirmação e Enfrentamento com risco), foi encontrada uma diferença significativa já entre avaliação intermediária e pós intervenção (diferenças entre as médias de 0,831 e $p=0,043$ ). Não foram encontradas diferenças estatisticamente significativas entre os escores da pós-intervenção e do follow-up, evidenciando a manutenção das aquisições.

A porcentagem de mudanças positivas e confiáveis, obtidas com o Método JT, considerando todo o grupo de participantes e os escores do IHS-Del-Prette, está apresentada a seguir na Tabela 2.

É possível verificar que houve oscilação positiva para mais de $60 \%$ dos participantes no Escore Total de habilidades sociais e nos Fatores Autoafirmação e enfrentamento com risco (F1) e Autoexposição a desconhecidos e situações novas (F4) ainda que nem todas fossem caracterizadas 


\section{INTERAÇÃO EM IT PSICOLOGIA}

Daniele Carolina Lopes, Denise Dascanio, Bárbara Carvalho Ferreira, Zilda Aparecida Pereira Del Prette e Almir Del Prette

\begin{tabular}{|c|c|c|c|}
\hline Indicadores (IHS-Del-Prette) & $\begin{array}{l}\text { OP } \\
(\%)\end{array}$ & $\begin{array}{l}\text { MPC } \\
(\%)\end{array}$ & $\begin{array}{l}\text { MNC } \\
(\%)\end{array}$ \\
\hline Escore Total (ET) & 77 & 20 & 3 \\
\hline $\begin{array}{l}\text { F1 - Autoafirmação e } \\
\text { Enfrentamento com risco }\end{array}$ & 74 & 51,4 & 12 \\
\hline $\begin{array}{l}\text { F2 - Autoafirmação na } \\
\text { expressão de afeto positivo }\end{array}$ & 61 & 11 & 5 \\
\hline $\begin{array}{l}\text { F3 - Conversação e desenvoltura } \\
\text { social }\end{array}$ & 26 & 14 & 3 \\
\hline $\begin{array}{l}\text { F4 - Autoexposição a } \\
\text { desconhecidos e situações } \\
\text { novas }\end{array}$ & 71 & 11 & 0 \\
\hline $\begin{array}{l}\text { F5 - Autocontrole da } \\
\text { agressividade }\end{array}$ & 40 & 3 & 8,5 \\
\hline
\end{tabular}

como mudança estatisticamente confiável (de melhora ou piora). Destaca-se a melhora estatisticamente confiável, portanto, atribuível ao programa, de mais de metade $(51,4 \%)$ dos participantes, nas habilidades do Fator 1 (Autoafirmação e enfrentamento com risco). Não obstante esses dados, houve oscilação no sentido de piora (mudança negativa confiável) em alguns indicadores, mas para no máximo $12 \%$ dos participantes no Fator 1. Ao analisar os participantes que não apresentaram melhoras significativas após a intervenção, observou-se que a maioria destes já apresentavam escores altos na avaliação de pré-teste no Escore total de habilidades sociais, configurando um efeito de teto que restringe a possibilidade de novas aquisições.

Outro indicador importante do impacto do programa foram os relatos espontâneos dos participantes, a cada sessão, sobre alterações na qualidade de suas interações cotidianas, conforme registradas no Diário de Campo. Elas se referiam a aquisições em habilidades de processo tais como auto-observação e observação de comportamento de outras pessoas, bem como a avaliações mais críticas sobre o próprio repertório, em termos de recursos e déficits de comportamentos. Outro conjunto de dados importantes foram os relatos escritos dos participantes, na sessão final de intervenção, quando solicitados a avaliarem o impacto da própria participação no PRODIP. Alguns desses relatos são reproduzidos a seguir.

Nunca tive um treinamento sobre as habilidades e aqui eu tive esse treinamento. Com o final do programa consigo perceber a evo- lução das minhas habilidades interpessoais e isso foi feito de forma agradável.

Uma experiência incrível em minha vida, que fez com que eu pensasse e passasse a agir de melhores maneiras, como eu não havia imaginado antes. Valeu a pena cada sessão, das quais aprendi coisas que certamente irei levar comigo por toda minha vida e que farão com que eu permaneça em constante aprendizado.

O programa é uma excelente maneira de se autoconhecer e também conhecer os métodos de monitorar isto. Para mim foi positivo, pois consigo entender melhor algumas reações das pessoas e também consigo me preparar melhor para como reagir.

Gostei muito do PRODIP, na verdade acho que foi a atividade de estágio que mais acrescentou conteúdos que possam me ajudar tanto profissionalmente quanto pessoalmente. A parte de colocar certas ações em prática me fez pensar e avaliar como eu me comporto. Espero continuar com esse tipo de reflexão (sobre o meu comportamento).

O PRODIP representou uma grande revolução na minha vida, pois mudou a percepção sobre vários acontecimentos ao meu redor. Além disso, me proporcionou um grande autoconhecimento, sobre minhas atitudes e forma de agir. E hoje posso considerar bem meIhor preparado para o mercado profissional.

\section{DISCUSSÃO}

O presente estudo reproduziu, em grande parte, os resultados obtidos por Z. Del Prette e Del Prette (2003), apontando para a potencialidade do PRODIP como estratégia de promoção de habilidades sociais relevantes, na transição do universitário para o mercado de trabalho. Destacam-se os ganhos no Escore Total de habilidades sociais, nas habilidades sociais de Autoafirmação e enfrentamento com risco (Fator 1) e de Autoexposição a desconhecidos e situações novas (Fator 4). Trata-se de um conjunto de habilidades sociais que podem ser consideradas relevantes para lidar com diferentes demandas atuais do mercado de trabalho, tais como trabalho em equipe, gestão de conflitos e relações de interdependência e interação direta, indireta e virtual (A. Del Prette \& Del Prette, 2003; Z. Del Prette \& Del Prette, 2003; Borchardt et al., 2009; Jenschke, 2003; Liebenberg \& Mathews, 2012; Moreno-Jimenez \& Camacho, 2014).

Além disto, pode-se observar que três meses após o término do programa, aqueles estudantes que melhoram com a intervenção mantiveram suas aquisições em habilidades sociais. Esse dado indica que as habilidades sociais desenvolvidas no PRODIP foram generalizadas para o ambiente de trabalho e para outros contextos diferentes da situação de estágio, com novos interlocutores, destacando a importância do 


\section{H. INTERACÃO EM * PSICOLOGIA}

Daniele Carolina Lopes, Denise Dascanio, Bárbara Carvalho Ferreira Zilda Aparecida Pereira Del Prette e Almir Del Prette programa em termos de sua validade social. Nesse sentido, o PRODIP foi considerado eficaz, uma vez que as condições de ensino planejadas, além de promoverem mudanças imediatas no repertório de habilidades sociais dos participantes, também foi eficiente por garantir a ocorrência e manutenção das habilidades sociais em situação natural (Cortegoso \& Coser, 2011).

Os resultados obtidos com a análise via Método JT mostraram que mais da metade dos participantes apresentou mudanças confiáveis (melhora), a maior parte delas nas habilidades de Autoafirmação e enfrentamento com risco (F1). Essa classe de habilidades sociais é requerida em situações interpessoais que envolvem a afirmação dos próprios direitos, preservando a autoestima, quando existe a possibilidade de reação indesejada do interlocutor, o que também é importante no contexto de trabalho. Essa subescala é constituída basicamente pelas habilidades denominadas assertivas. 0 tópico assertividade vem se disseminando no âmbito das organizações como uma competência relevante buscada pelos gestores, inclusive em anúncios que especificam requisitos para o preenchimento de algumas funções e empregos ("Analista de recursos humanos", 2012; "Gerente administrativo financeiro", 2012; "Suave poder", 2013).

0 conjunto dos resultados, mostrando oscilações positivas para a maioria dos participantes, com parte delas estatisticamente confiáveis, se assemelha aos resultados obtidos por outros estudos, ainda que estes não tenham utilizado o Método JT (Bolsoni-Silva et al., 2009; Boas et al., 2005; A. Del Prette \& Del Prette, 2003; Del Prette et al., 1999; Magalhães \& Murta, 2003; Pacheco \& Rangé, 2006). Os dados de não meIhora, ou mesmo de piora de desempenho, alertam para a possibilidade de o programa não ser efetivo para alguns tipos de necessidades, características ou dificuldades dos participantes, tal como aconteceu em outros estudos que não alcançaram melhora para todos os participantes (Bolsoni-Silva et al., 2009; Boas et al., 2005). As observações informais ao longo das sessões, registradas no Diário de Campo, sugerem que os participantes aprenderam a observar e a descrever melhor seus comportamentos, adotando uma postura mais crítica ao avaliarem o próprio desempenho. É possível que isso explique alguns casos de queda de escore em habilidades sociais, especialmente em participantes com alto escore inicial, por exemplo, aquelas relacionadas ao Fator 5 - Autocontrole da agressividade. Essa hipótese também é aventada em outros estudos (Boas et al., 2005; A. Del Prette, \& Del Prette, 2003).

Não obstante suas possíveis contribuições empíricas, os autores reconhecem limitações do presente estudo. Uma delas foi ter se baseado principalmente em dados de relato, tal como os obtidos com o Inventário de Habilidades Sociais (IHS-Del-Prette - Z. Del Prette \& Del Prette, 2001) e com relatos assistemáticos ao longo das sessões e ao final do programa. Ainda que a observação direta do desempenho dos participantes tenha guiado todo o planejamento do programa, por vezes indicando melhores resultados do que a autoavaliação, não foi realizada uma coleta regular e sistematizada desses desempenhos. Estudos futuros poderão incluir, em cada momento de avaliação, o uso de registros observacionais, como feito por Pereira-Guizzo, Del Prette e Del Prette (2012), por meio de situações estruturadas filmadas, como também por observações diretas nas sessões de forma mais sistematizada do que aconteceu apenas com os diários de campo. Um aprimoramento também importante na avaliação do PRODIP seria a participação de supervisores das empresas ou de pessoas com quem os participantes convivem, para uma avaliação externa dos participantes, o que certamente traria dados adicionais particularmente relevantes quanto à validade externa do programa.

No que se refere à validade interna, uma limitação do presente estudo foi a ausência de grupo controle, o que não permite afirmar, com segurança, que os resultados alcançados são exclusivamente devidos à intervenção e não a variáveis estranhas (Cozby, 2003). Essa limitação é, em parte, amenizada pela similaridade da replicação das edições consecutivas do programa. Estudos futuros poderiam combinar outros instrumentos e procedimentos de avaliação e utilizar delineamentos experimentais mais controlados, por exemplo, com grupo controle (Rocha, Del Prette \& Del Prette, 2013) e/ou placebo (Braz, Del Prette \& Del Prette, 2011).

Considerando os estudos sobre programas de promoção de habilidades sociais para estudantes que não cursaram Psicologia, este estudo apresenta semelhanças com outros programas, em termos de procedimentos de intervenção, com uso de vivências, tarefas de casa, role-play em sessão (Bolsoni-Silva et al., 2009; Del Prette \& Del Prette, 2003). Em contrapartida, não foi focado apenas em uma classe de habilidades sociais, como o de Falcone (1999) e o de Kestenberg e Falcone (2011), e não teve como critério de seleção apenas participantes com queixas de dificuldades interpessoais e/ou de adaptação à Universidade (Boas et al., 2005; Bolsoni-Silva et al., 2009), pelo contrário, o específico deste trabalho envolveu estudantes universitários que já haviam sido pré-selecionados em relação a algumas habilidades para o programa de estágio envolvendo capacitação em liderança denominado PROLÍDER (Gerolamo \& Gambi, 2013) conjuntamente com as empresas pelas quais seriam contratados para estagiar, ou seja, se configuravam como participantes em vias de transição para o mercado de trabalho.

Ainda que com delineamento pré e pós-teste, o presente 


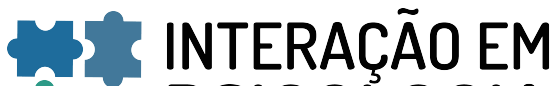 in PSICOLOGIA}

estudo acrescenta evidências favoráveis ao PRODIP, junto a universitários de Ciências Exatas, em particular devido às avaliações sucessivas, que permitiram identificar a aquisição e manutenção de habilidades sociais relevantes para enfrentarem as atuais demandas do mercado de trabalho. Além das questões de pesquisa já referidas, tais programas possuem implicações sociais que já não podem ser ignoradas.

O notável avanço tecnológico, inclusive e principalmente nos recursos de intercâmbio entre estados, organizações e pessoas, longe de prescindir de gestões e desenvolvimento de comunicação interpessoal, vem requerendo, cada vez mais, melhor preparo funcional do trabalhador em diferentes níveis de atuação. Isso se reflete em um mercado de trabalho cada vez mais exigente quanto à formação profissional. Essa situação se desdobra em uma pressão sobre a Universidade em seu compromisso com a preparação das novas gerações. A competência técnica, tradicionalmente esperada como resultado de anos de estudo, deve, na atualidade, ser acompanhada da competência social (Z. Del Prette \& Del Prette, 2003). Nesse sentido, o PRODIP e outros programas voltados para melhorar a qualidade das relações interpessoais, podem constituir alternativas importantes como forma de complemento da formação profissional, reduzindo, nas organizações, em médio e longo prazo, um conjunto de demandas de treinamento de pessoal.

\section{CONTRIBUIÇÃO DE CADA AUTOR}

D. C. L., D. D. e B. C. F contribuíram para a conceitualização, investigação, análise formal dos dados, tabulação dos dados, redação - preparação do rascunho original e visualização. D. C. L., D. D., B. C. F., Z. A. P. D. P e A. D. P. compartilharam a contribuição quanto à metodologia $e$ redação - revisão e edição. Z. A. P. D. P e A. D. P. administraram o projeto, supervisionaram e validaram.

\section{DECLARAÇÃO DE CONFLITOS DE INTERESSES}

Os autores declaram que não há conflitos de interesses no presente artigo.

\section{REFERÊNCIAS}

Aguiar, A. A. R., Aguiar, R. G., \& Del Prette, Z. A. P. (2009). Calculando a significância clínica e o Índice de Mudança Confiável em pesquisa-intervenção. São Carlos: EDUFSCar.

Analista de recursos humanos (2012). Manager online.

Argyle, M. (1994). Psicologia del comportamiento interpersonal. Madrid: Alianza Universidad. (Trabalho original publicado em 1967).
Daniele Carolina Lopes, Denise Dascanio, Bárbara Carvalho Ferreira Zilda Aparecida Pereira Del Prette e Almir Del Prette

Bandeira, M., \& Quaglia, M. A. C. (2005). Habilidades sociais de estudantes universitários: identificação de situações sociais significativas. Interação em Psicologia, 9(1), 45-55.

Bandeira, M., Costa, M. N., Del Prette, Z. A. P., Del Prette, A., \& Gerk-Carneiro, E. (2000). Qualidades psicométricas do Inventário de Habilidades Sociais (IHS): Estudo sobre a estabilidade temporal e a validade concomitante. Estudos de Psicologia, 5(2), 401-419.

Boas, A. C. V. V., Silveira, F. F., \& Bolsoni-Silva, A. T. (2005). Descrição de efeitos de um procedimento de intervenção em grupo com universitários: um estudo piloto. Interação em Psicologia, 9(2), 312-330.

Bolsoni-Silva, A. T., \& Loureiro, S. R. (2016). Validação do Questionário de Avaliação de Habilidades Sociais, Comportamentos, Contextos para Universitários. Psicologia: Teoria e Pesquisa, 32(2), 1-10.

Bolsoni-Silva, A. T., Del Prette, Z. A. P., Del Prette, G., Montagner, A. R., Bandeira, M., \& Del Prette, A. (2006). Habilidades sociais no Brasil: Uma análise dos estudos publicados em periódicos. Em M. Bandeira, Z. A. P. Del Prette \& A. Del Prette (Eds.), Estudos sobre habilidades sociais e relacionamento interpessoal (pp. 1-45). São Paulo: Casa do Psicólogo.

Bolsoni-Silva, A. T., Leme, V. B. R, Lima, A. M. A., Costa-Júnior, F. M., \& Correia, M. R. (2009). Avaliação de um treinamento de habilidades sociais (THS) com universitários e recém formados. Interação em Psicologia, 13(2), 241-251.

Bolsoni-Silva, A. T., Loureiro, S. R., Rosa, C. F., \& Oliveira, M. C. F. A. (2010). Caracterização das habilidades sociais em universitários. Contextos Clínicos, 3(1), 62-75.

Borchardt, M., Vaccaro, G. L. R., Azevedo, D., \& Ponte Jr., J. (2009). O perfil do engenheiro de produção: A visão de empresas da região metropolitana de Porto Alegre. Produção, 19(2), 230-248.

Botomé, S. P. (2010). A quem, nós psicólogos, servimos de fato?. Em O. H. Yamamoto, \& A. L. F. Costa. (Eds.), Escritos sobre a profissão de psicólogo no Brasil (pp.169-202). Natal: EDUFRN.

Braz, A. C., Del Prette, Z. A. P., \& Del Prette, A. (2011). Assertive social skills training for the elderly. Behavioral Psychology/ Psicologia Conductual, 19(2), 373-387.

Carneiro, A. A., \& Teixeira, C. M. (2011). Avaliação de habilidades sociais em alunos de graduação em psicologia da Universidade Federal do Maranhão. Psicologia, Ensino \& Formação, 2(1), 43-56.

Cortegoso, A. L., \& Coser, D. S. (2011). Elaboração de Programas de Ensino: Material autoinstrutivo. São Carlos: EDUFSCar.

Cozby, P. C. (2003). Métodos de pesquisa em ciências do comportamento. São Paulo: Atlas. 


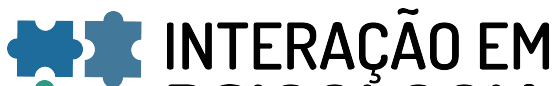 ET PSICOLOGIA}

Del Prette, A., \& Del Prette, Z. A. P. (2001). Psicologia das relações interpessoais: vivências para o trabalho em grupo. Petrópolis: Vozes.

Del Prette, A., \& Del Prette, Z. A. P. (2003). No contexto da travessia para o ambiente de trabalho: treinamento em habilidades sociais com universitários. Estudos de Psicologia (Natal), 8(3), 413-420.

Del Prette, A., \& Del Prette, Z. A. P. (2005). A importância das tarefas de casa como procedimento para a generalização e validação do Treinamento de Habilidades Sociais. Em H. Guilhardi, \& N. C. Aguirre (Eds.), Primeiros passos em Análise do Comportamento e Cognição (pp. 67-74). Santo André: ESETec.

Del Prette, A., \& Del Prette, Z. A. P. (2010). Programa vivencial de habilidades sociais: Características sob a perspectiva da análise do comportamento. Em M. R. Garcia, P. Abreu, E. N. P. de Cillo, P. B. Faleiros, \& P. P. Queiroz (Eds.), Comportamento e Cognição: Terapia Comportamental e Cognitiva (pp. 127-139). Santo André: ESETec.

Del Prette, A. (1978). O treino assertivo na formação do psicólogo. Arquivos Brasileiros de Psicologia, 30, 53-55.

Del Prette, A., Del Prette, Z. A. P., \& Barreto, M. C. M. (1999). Habilidades sociales en la formación del psicólogo: Analisis de un programa de intervención. Psicología Conductual, 7(1), 27-47.

Del Prette, Z. A. P., \& Del Prette, A. (2001). Inventário de habilidades sociais (IHS-Del-Prette): Manual de aplicação, apuração e interpretação. São Paulo: Casa do Psicólogo.

Del Prette, Z. A. P., \& Del Prette, A. (2003). Desenvolvimento interpessoal: Uma questão pendente no ensino universitário. Em E. Mercuri, \& S. A. J. Polydoro (Eds.), Estudante universitário: Características e experiências de formação (pp. 105-128). Taubaté: Cabral Editora e Livraria Universitária.

Del Prette, Z. A. P., Del Prette, A. \& Barreto, M. C. M. (1998), Análise de um Inventário de Habilidades Sociais (IHS) em uma amostra de universitários. Psicologia: Teoria e Pesquisa, 14(3), 219-228.

Del Prette, Z. A. P., Del Prette, A., Barreto, M. C. M., Bandeira, M., Rios-Saldaña, M. R., Ulian, A. L. A. O., Gerk-Carneiro, E., Falcone, E. M. O., \& Villa, M. B. (2004). Habilidades sociais de estudantes de psicologia: um estudo multicêntrico. Psicologia: Reflexão e Crítica, 17(3), 341-350.

Del Prette, Z. A. P., Del Prette, A., Rios-Saldaña, M. R., Caballo, V. E., Bandeira, M., Falcone, E. M. O., Gerk-Carneiro, E., Ulian, A. L. A. O., \& Barreto, M. C. M. (2004). Un estudio transcultural con estudiantes de psicología: Habilidades sociales de brasileños, mexicanos y españoles. Alternativas en Psicologia (México), IX (10), 69-82.

Falcone, E. M. O. (1999). Avaliação de um programa de treinamento de empatia com universitários. Revista Brasileira de Terapia Comportamental e Cognitiva, 1(1), 23-32.
Daniele Carolina Lopes, Denise Dascanio, Bárbara Carvalho Ferreira Zilda Aparecida Pereira Del Prette e Almir Del Prette

Fang, N. (2012). Improving Engineering Students' Technical and Professional Skills Through Project-Based Active and Collaborative Learning, International Journal of Engineering Education, 28(1), 26-36.

Furtado, E. S., Falcone, E. M. O., \& Clark, C. (2003). Avaliação do estresse e das habilidades

Gerente Administrativo Financeiro. (2012). Elancers. Recuperada em 17 de dezembro, 2012 de http://www.elancers.net/spl_v3/vinculo_vagas.asp?par1 $=6974$ DE58506B 907B88FAE862505C8536

Gerolamo, M. C., \& Gambi, L. N. (2013). How Can Engineering Students Learn Leadership Skills? The Leadership Development Program in Engineering (PROLIDER) at EESC-USP, Brazil. International Journal of Engineering Education, 29(5), 1172-1183.

Gil, F. (1998). Habilidades sociales en el trabajo y en las organizaciones. Em F. Gil e J. M. León (Eds.), Habilidades sociales: Teoría, investigácion e intervención (pp. 197-202). Madrid: Síntesis Psicologia.

Gomes, G., \& Soares, A. B. (2013). Inteligência, habilidades sociais e expectativas acadêmicas no desempenho de estudantes universitários. Psicologia: Reflexão e Crítica, 26(4), 780-789.

Gondim, S. M. G. (2002). Perfil profissional e mercado de trabalho: Relação com a formação acadêmica pela perspectiva de estudantes universitários. Estudos de Psicologia, 7(2), 299-309.

Índice Brasileiro de Opinião Pública e Estatística - IBOPE (2005). Critério de Classificação Econômica Brasil.

International Labour Organization - ILO (2006). Changing patterns in the world of work. Genebra: Autor.

Jablokow, K. W. (2008). Developing Problem Solving Leadership: A Cognitive Approach, International Journal of Engineer Education, 24(5), 936-954.

Jacobson, N. S., \& Truax, P. (1991). Clinical significance: A statistical approach to defining meaningful change in psychotherapy research. Journal of Consulting and Clinical Psychology, 59, 12-19.

Jenschke, B. (2003). A cooperação internacional: Desafios e necessidades da orientação e do aconselhamento em face das mudanças mundiais no trabalho e na sociedade. Revista Brasileira de Orientação Profissional, 4(1/2), 35-55.

Kestenberg, C. C. F., \& Falcone, E. M. O. (2011). Programa de promoção da empatia para graduandos em enfermagem. Em A. Del Prette, \& Z. A. P. Del Prette (Eds.), Habilidades sociais: Intervenções efetivas em grupo (pp. 115-143). São Paulo: Casa do Psicólogo.

Kienen, N., Kubo, O. M., \& Botomé, S. P. (2013). Ensino programado e programação de condições para o desenvolvimento de comportamentos: alguns aspectos no desenvolvimento de um campo de atuação do psicólogo. Acta Comportamentalia, 21(4), 481-494. 


\section{-4: INTERACÃO EM LF PSICOLOGIA}

Kubo, O. M., \& Botomé, S. P. (2003). A transformação do conhecimento em comportamentos profissionais na formação do psicólogo: as possibilidades das diretrizes curriculares. Em M. Z. S. Brandão, F. C. S. Conte, F. S. Brandão, Y. K. Ingerman, Y. K., C. B. Moura, V. M. Silva, \& S. M. Oliane (Eds.), Sobre Comportamento e Cognição (vol. 11, pp. 483-496). Santo André, SP: ESETec Editores Associados.

Liebenberg, L., \& Mathews, E. H. (2012). Integrating innovation skills in an introductory engineering design-build course. International Journal of Technology and Design Education, $22(1), 93-113$

Magalhães, P. P., \& Murta, S. G. (2003). Treinamento de habilidades sociais de estudantes de psicologia: um estudo pré-experimental. Temas em Psicologia, 11(1), 28-37.

Marqués, P., Farrerons, L., Arian, N., \& Quiroga, E. (2012). Las relaciones informales: un valor añadido en la gestión de personas. Revista electrónica Enfermería Global, 26, 310323.

Moreno-Jiménez, B. Garrosa, E. H. \& Rodríguez-Carvajal, R. (2013). Las organizaciones saludables. Em B. Moreno-Jimenez \& E. Garrosa Hernández (Eds.), Salud laboral. Riesgos laborales psicosociales y bienestar laboral (pp. 295-312). Madrid: Pirámide.

Moreno-Jiménez, B., \& Camacho, A. A. (2014). Habilidades sociales para las nuevas organizaciones. Behavioral Psychology/Psicología Conductual, 22(3), 587-604.

Mourão, L., \& Puente-Palacios, K. E. (2006). Formação profissional. Em J. E. Borges-Andrade, G. S. Abbad \& L. Mourão (Eds.), Treinamento, Desenvolvimento e Educação em Organizações e Trabalho: fundamentos para a gestão de pessoas (pp. 41-64). Porto Alegre: Artmed.

Pacheco, P., \& Rangé, B. (2006). Desenvolvimento de habilidades sociais em graduandos de Psicologia. Em M. Bandeira, Z. A. P. Del Prette, \& A. Del Prette (Eds.), Estudos sobre habilidades sociais e relacionamento interpessoal (pp. 199-216). São Paulo: Casa do Psicólogo.
Daniele Carolina Lopes, Denise Dascanio, Bárbara Carvalho Ferreira Zilda Aparecida Pereira Del Prette e Almir Del Prette

Pereira-Guizzo, C. S., Del Prette, A., \& Del Prette, Z. A. P. (2012). Evatuation of a professional social skills program for unemployed people with physical disability. Psicologia: Reflexão e Crítica, 25(4), 265-274.

Ponsa, P., Manresa-Yee, C., Arellano, D., Gomez, J., \& Perez, A. (2012). Human-Centred Design in Engineering Curricula. International Journal of Engineering Education, 28(4), 767-777.

Prytz Nilsson, N., Suarez, A. B., \& Olaz, F. O. (2009, outubro). Estudio bibliometrico de las publicaciones científicas sobre el área de habilidades sociales em América Latina. Actas de VI Congreso Iberoamericano de Psicología Clínica y de la Salud (APICSA). Santiago de Chile, p. 139-140.

Ribeiro, D., \& Bolsoni-Silva, A. T. (2011). Potencialidades e dificuldades interpessoais de universitários: estudo de caracterização. Revista Acta Comportamentalia, 19(2), 205-224.

Rocha, M. M., Del Prette, Z. A. P., \& Del Prette, A. (2013). Avaliação de um Programa de Habilidades Sociais Educativas para mães de crianças com TDAH. Acta Comportamentalia, 21(3), 359-375.

Silva, B. M. B., \& Teixeira, M. A. P. (2012). Autopercepção de competências transversais de trabalho em universitários: construção de um instrumento. Estudos de Psicologia, 17(2), 199-206.

Soares, A. B., Poubel, L. N., \& Mello, T. V. S. (2009). Habilidades sociais e adaptação acadêmica: Um estudo comparativo em instituições de ensino público e privado. Aletheia, $29,27-42$.

Suave poder: Habilidades sociais ganham importância nos processos seletivos das empresas (03 de fevereiro, 2013) Jornal Folha de São Paulo, pp. F1.

Recebido em: 14/04/2014 Primeira decisão editorial em: 18/01/2017 Aceito em: 13/03/2017

10 Critério Brasil de Classificação Econômica Brasil (IBOPE/ABEP, 2005) é um instrumento que avalia o nível socioeconômico das famílias com base no poder aquisitivo, número de bens de consumo duráveis, presença de empregada doméstica e grau de instrução do chefe da família 